\title{
Flow Cytometric Analysis of Depletion and Recovery Kinetics of T Cell Subsets in Rats After Total-Body- Irradiation: Footprints of Treg-Augmented Immunosuppression
}

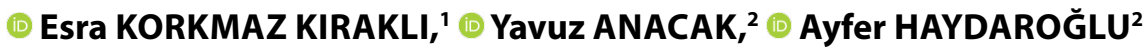 \\ 'Department of Radiation Oncology, Dr. Suat Seren Chest Diseases and Thoracic Surgery Training and Research Hospital, Radiation \\ Oncology, İzmir-Turkey \\ ${ }^{2}$ Department of Radiation Oncology, Ege University School of Medicine, İzmir-Turkey
}

\begin{abstract}
OBJECTIVE
Total-body-irradiation (TBI) causes significant immunosuppression, but different lymphocyte subsets have various radiosensitivities. Regulatory T (Treg) cells, which are crucial for self-tolerance and are potent suppressors of antitumor immunity are found to be resistant to radiotherapy (RT) compared with Thelper (Th) cells and Cytotoxic-T lymphocytes (CTL) in both in-vivo and in-vitro studies, but the data on this subject is relatively scarce. Besides, recent developments in the context of combination of immunotherapy with RT compelled us to revisit the concept of radiation-induced quantitative and functional changes in lymphocyte subsets by flow cytometry using animal models with the aim of transitioning the findings to clinical studies.
\end{abstract}

\section{METHODS}

Twenty-three Swiss albino rats were exposed to TBI at a single fraction of $5 \mathrm{~Gy}$. Immediately prior to irradiation, at time points of 1 day and 7 and 14 days post-TBI, flow cytometric analyses were performed.

\section{RESULTS}

There has been statistically significant decrease in all $\mathrm{T}$ lymphocyte subsets at 1,7 and 14 days post-TBI. The decrease in Th subset was more pronounced compared to CTL. Baseline CD4+/CD8+ ratio was 0.85 which significantly decreased to 0.291 day post-TBI, then increased steadily in subsequent measurements and reached near normal. The number of Treg cells markedly declined to $6.5 \%$ of baseline value one day after TBI, and then steadily increased during the follow-up. By the end of 14 days, it reached half of its baseline value.

\section{CONCLUSION}

Radiation-induced immunosuppression may be explained not only by the decrease in lymphocyte cell number but also by the relative increase in Treg cell number because the higher regenerative capacity may present an additional role.

Keywords: Immune dysfunction; T lymphocytes; total-body-irradiation. Copyright $\odot$ 2018, Turkish Society for Radiation Oncology

\section{Introduction}

Occupational, environmental, accidental, or therapeutic exposure to ionizing radiation have major impacts on human health and may result in defects in the hematopoietic and immune systems.[1] Lymphocytes have the highest cell turnover in mammalians, which makes them extremely radiosensitive.[2,3] Although
Received: May 28, 2018

Accepted: June 03, 2018

Online: July 05, 2018

Accessible online at:

www.onkder.org
Dr. Esra KORKMAZ KIRAKLI

Dr. Suat Seren Göğüs Hastalıkları ve

Cerrrahisi Eğitim ve Araştırma Hastanesi,

Radyasyon Onkolojisi,

İzmir-Turkey

E-mail: esrakirakli@gmail.com 
the chromosome-aberration assay remains the "gold standard" for early-response accident biodosimetry and dose assessment, the lymphocytes are accepted as the most efficient practical laboratory tool for estimating exposed dose in population monitoring and they enable prompt commencement of medical intervention in case of nuclear accidents.[4,5]

TBI causes significant immunosuppression, but different lymphocyte subsets have various radiosensitivities; B cells are the most sensitive ones and Natural killer cells are the most resistant ones. T helper/effector cells (Th) are responsible for the regulation of immune system and are more radiosensitive than $\mathrm{T}$ cytotoxic/suppressor cells (CTL), which directly destroy target cells. [6] T regulatory (Treg) cells are crucial for self-tolerance and are the potent suppressors of antitumor immunity. $[7,8]$ They are found to be resistant to IR compared with Th and CTL cells in both in vivo and in-vitro studies, but there is limited data on this.[7,9-11] Developments in flow cytometry provide us with a detailed analysis of lymphocyte subsets using monoclonal antibodies (MAbs) specific to lymphocyte differentiation antigens. [12] In addition, recent developments and rising questions in the context of combining immunotherapy with RT compelled us to revisit the concept of radiation-induced quantitative and functional changes in lymphocyte subsets by flow cytometry using animal models with the aim of transitioning the findings to clinical studies.

\section{Materials and Methods}

Twenty-three Swiss albino rats of both sexes weighing $200-250 \mathrm{~g}$ at $4-8$ weeks of age were evaluated. Animals were housed in a dedicated animal room maintained at $22^{\circ} \mathrm{C} \pm 2^{\circ} \mathrm{C}$ and a relative humidity of $50 \% \pm 20 \%$. Animals remained on 12:12-h light and dark cycles with free access to food and water.

\section{Experimental Design}

Immediately prior to irradiation, $1 \mathrm{cc}$. heparinized blood sample was drawn via cardiac puncture under superficial anesthesia. Next, flow cytometric analysis was performed within two hours by the following fluorescent labeled MAbs designed specifically for rats: CD3-FITC (Fluorescein isothiocyanate), CD4-PE (phycoerythrin), CD8-A PE, CD25 IL-2, Mouse Anti-Rat CD3-IF4, and Mouse Anti-Rat CD25-OX39 at the immunology laboratory. First, antibodies were added and after waiting for 15 min under room temperature and darkness, erythrocytes were lysed using FACS Lysing solution (Beckton and Dickinson, San Jose, CA), cen- trifuged, and washed with Phosphate Buffer Solution. After washing, flow cytometric analysis was performed using FACSCAN flow cytometry system (Beckton and Dickinson, San Jose, CA) and read on simulset software program. Using CD3-FTIC, lymphocytes were gated by isotope match negative control antibodies and were differentiated into FITC and PE channels. Analysis was performed by assaying 15,000 spheres per tube.

Next, the rats were exposed to TBI under ketamine anesthesia $(75 \mathrm{mg} / \mathrm{kg}-\mathrm{IM})$ at a single fraction of $5 \mathrm{~Gy}$. Irradiation (IR) was performed using $\mathrm{Co}_{60} \gamma$-rays and administered to a midline tissue. Following this, the IR rats were taken back to their home cages. The peripheral blood samples were redrawn at time points of 1 day (acute period) and 7 and 14 days (latent period) post-TBI and flow cytometric analyses were performed again. In addition, complete blood counts with differentials were performed in pediatric hematology laboratory using a clinical hematology analyzer.

$\mathrm{CD}^{+} \mathrm{CD} 19^{-}$represent $\mathrm{T}$ lymphocytes, $\mathrm{CD}^{+}{ }^{+} \mathrm{CD} 4^{+}$ represent $\mathrm{T}$ helper/inducer cells (Th), $\mathrm{CD}^{+} \mathrm{CD}^{+}$ represent $\mathrm{T}$ cytotoxic/suppressor cell (CTL), and $\mathrm{CD} 4^{+} \mathrm{CD} 25^{+}$represent Treg cells. [13]

The ratio of lymphocyte subsets are calculated by dividing the total $\mathrm{T}$ lymphocyte count by absolute lymphocyte subset numbers. The lymphocyte subset depletion and recovery kinetics by time were analyzed.

None of the animals were euthanized.

\section{Statistical Analysis}

Statistical analysis was performed using SPSS 21.0 software (SPSS Inc., Chicago, IL). Continuous variables were expressed as mean \pm standard deviation and categorical variables were expressed as $\mathrm{n}(\%)$. Comparisons were done using Repeated Measures ANOVA or Fischer's exact test where appropriate. A p value of $<0.05$ was considered statistically significant.

\section{Results}

White blood cell and lymphocytes counts in the peripheral blood at baseline and at 1, 7, and 14 days after TBI are presented in Table 1 and Figure 1.

At 1,7 , and 14 days after TBI, a statistically significant decrease was noted in all $\mathrm{T}$ lymphocyte subsets compared with the baseline value both in terms of absolute number and ratio (Table 2-3 and Fig. 2). The decrease in Th subset was more pronounced compared with that in CTL.

Baseline $\mathrm{CD}^{+} / \mathrm{CD}^{+}$ratio was 0.85 . This ratio significantly decreased to 0.29 of the baseline value one 
Table 1 Mean absolute leucocyte and lymphocyte levels at baseline and 1, 7, and 14 days after Total-Body-Irradiation (TBI)

\begin{tabular}{lcccr} 
& Baseline & 1 day after TBI & 7 days after TBI & 14 days after TBI \\
\hline Leucocyte \# & $21376 \pm 6358$ & $6333 \pm 5149(p<0.01)$ & $6533 \pm 6050(p<0.01)$ & $4823 \pm 2437(p<0.01)$ \\
Lymphocyte \# & $16618 \pm 5255$ & $2614 \pm 2268(p<0.01)$ & $3403 \pm 2607(p<0.01)$ & $3285 \pm 1903(p<0.01)$ \\
\hline
\end{tabular}

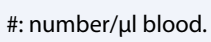

Table 2 Mean lymphocyte subset ratios at baseline and 1, 7, and 14 days after Total-Body-Irradiation (TBI) according to flow cytometric analysis

\begin{tabular}{lcccc} 
Subtype & Baseline & 1 day after TBI & 7 days after TBI & 14 days after TBI \\
\hline T lymphocyte & $61 \% \pm 14$ & $48 \% \pm 19(\mathrm{p}<0.01)$ & $62 \% \pm 25(\mathrm{p}<0.01)$ & $55 \% \pm 22(\mathrm{p}<0.01)$ \\
Thelper & $27 \% \pm 5$ & $10 \% \pm 5(\mathrm{p}<0.01)$ & $24 \% \pm 10(\mathrm{p}<0.01)$ & $21 \% \pm 14(\mathrm{p}<0.01)$ \\
CTL & $38 \% \pm 14$ & $40 \% \pm 17(\mathrm{p}<0.01)$ & $42 \% \pm 18(\mathrm{p}<0.01)$ & $35 \% \pm 16(\mathrm{p}<0.01)$ \\
\hline
\end{tabular}

Table 3 Mean absolute lymphocyte subset levels/ $\mu$ l blood at baseline and 1, 7, and 14 days after Total-Body-Irradiation (TBI) according to flow cytometric analysis

\begin{tabular}{lcccc} 
Subtype & Baseline & $\mathbf{1}$ day after TBI & $\mathbf{7}$ days after TBI & $\mathbf{1 4}$ days after TBI \\
\hline T lymphocyte & $10473 \pm 4853$ & $1525 \pm 1607(p<0.01)$ & $1899 \pm 1278(p<0.01)$ & $2092 \pm 1564(p<0.01)$ \\
T hepler & $4696 \pm 1354$ & $274 \pm 369(p<0.01)$ & $737 \pm(p<0.01)$ & $799 \pm 625(p<0.01)$ \\
CTL & $6448 \pm 33812$ & $1271 \pm 1288(p<0.01)$ & $1278 \pm 836(p<0.01)$ & $1373 \pm 1176(p<0.01)$
\end{tabular}

Table 4 The changes in CD4+/ CD8+ ratio at baseline and 1, 7, 14 days after Total-Body-Irradiation (TBI) according to flow cytometric analysis

\begin{tabular}{lcccc} 
& Baseline & 1 day after TBI & 7 days after TBI & $\mathbf{1 4}$ days after TBI \\
\hline CD4+/CD8+ & $0.85 \pm 0.37$ & $0.29 \pm 0.18(p<0.01)$ & $0.64 \pm 0.28(p<0.01)$ & $0.69 \pm 0.46(p<0.01)$ \\
\hline
\end{tabular}
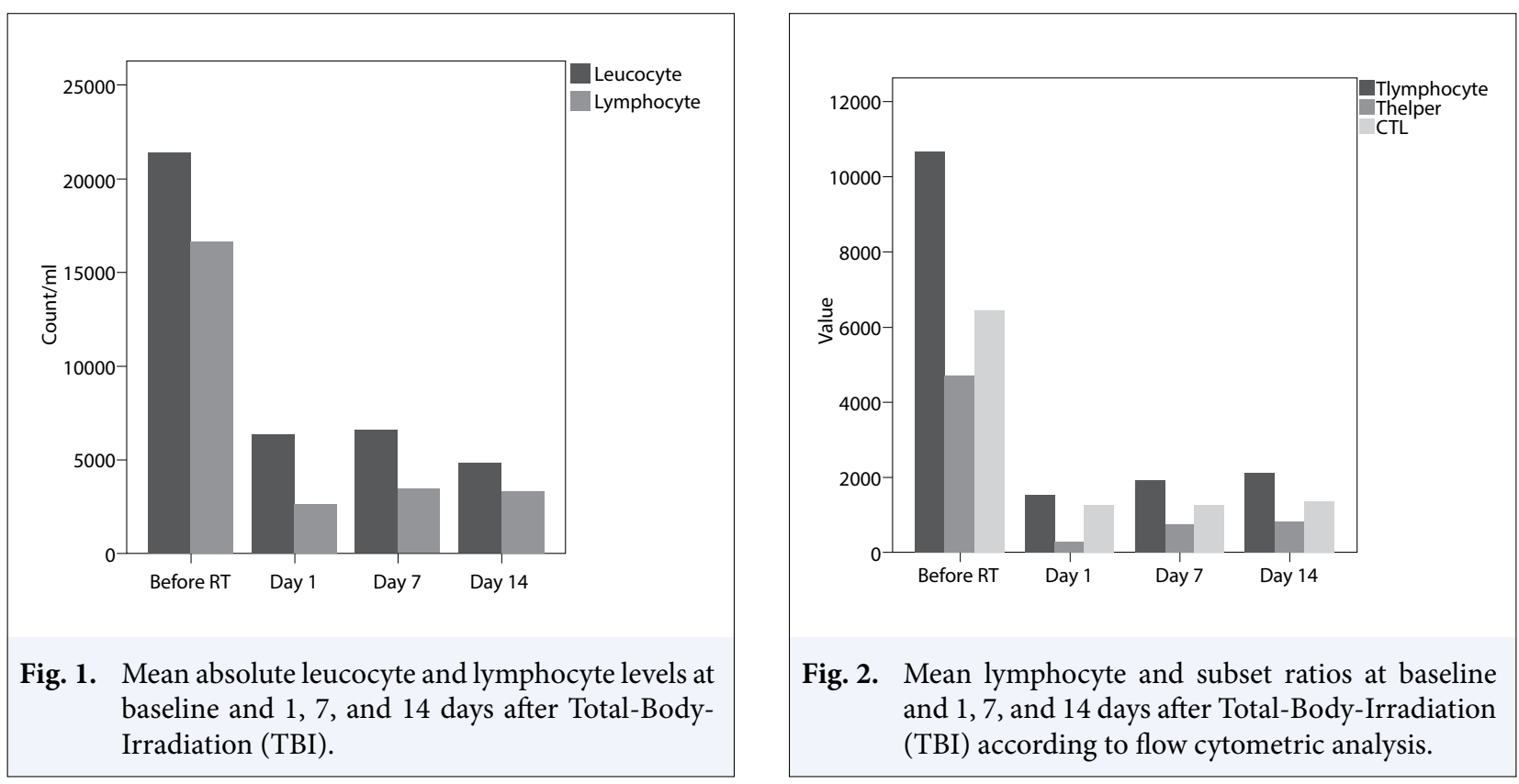


\begin{tabular}{|c|c|c|c|c|}
\hline \multirow[t]{2}{*}{ Table 5} & \multicolumn{4}{|c|}{$\begin{array}{l}\text { Mean absolute number of Treg cells/ } \mu \text { l blood at baseline and 1,7, } 14 \text { days after Total-Body-Irradiation (TBI) ac- } \\
\text { cording to flow cytometric analysis }\end{array}$} \\
\hline & Baseline & 1 day after TBI & 7 days after TBI & 14 days after TBI \\
\hline Treg & $153 \pm 104$ & $10 \pm 13(p<0.01)$ & $48 \pm 38(p<0.01)$ & $70 \pm 117(p<0.01)$ \\
\hline
\end{tabular}

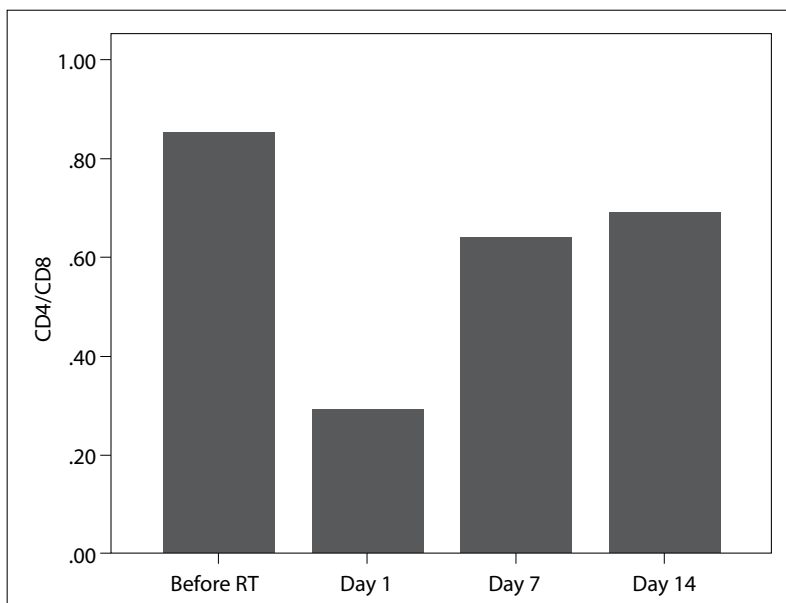

Fig. 3. $\mathrm{CD}^{+} / \mathrm{CD}^{+}$ratio at baseline and $1,7,14$ days after Total-Body-Irradiation (TBI) according to flow cytometric analysis.

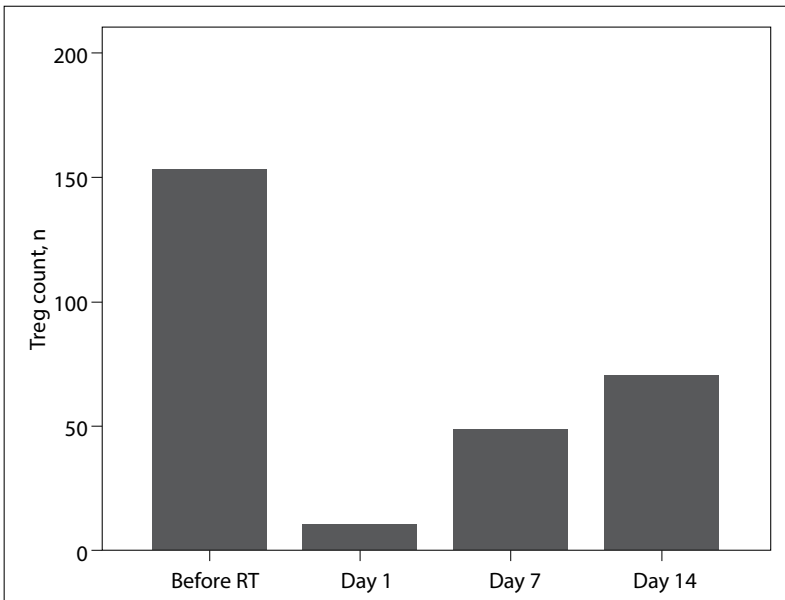

Fig. 4. Mean absolute number of Treg cells/ $\mu$ blood at baseline and 1, 7, 14 days after Total-Body-Irradiation (TBI) according to flow cytometric analysis.

day after TBI, then steadily increased in subsequent measurements and reached a near normal value (Table 4 and Fig. 3).

Treg lymphocyte level markedly declined to $6.5 \%$ of baseline value one day after TBI, and then steadily increased during follow-up. By the end of 14 days, it reached half of its baseline value (Table 5 and Fig. 4).

\section{Discussion}

Our study showed that a statistically significant decrease in absolute leucocyte and lymphocyte subset number levels in peripheral blood was observed as early as one day after exposure to 5 Gy TBI; lymphocyte subsets have different radiosensitivities and monitoring $\mathrm{T}$ lymphocyte subset depletion and recovery kinetics as a biodosimetry has potential utility for predicting the dose, timing, and outcome of radiation exposure. Also, we have found that Treg cell recovery was faster than that of Th and CTL. This finding may contribute to Treg-augmented immunosuppression in addition to direct immunosuppressive effects of ionizing radiation.

The mean Lethal Dose $\left(\mathrm{LD}_{50}\right)$ is defined as a dose that results in $50 \%$ mortality in a population within 30-60 days after TBI.[14] $\mathrm{LD}_{50}$ is accepted at 7.6-7.8 Gy in rats. [15] Because lymphocytes are extremely sensitive to IR, doses close to $\mathrm{LD}_{50}$ may cause significant lymphocyte depletion $(<200$ cells $/ \mathrm{mm})$; as a result their utility in assessing the exposed dose may be challenging.[16] Therefore, TBI dose of 5 Gy was preferred in this study since it is a sublethal dose.

Peripheral blood samples were collected one day after TBI because after 24-48 hours of IR exposure, a predictable decrease in absolute lymphocyte counts occurs that leads to the use of lymphocyte depletion kinetics as a part of biodosimetric model in case of radiation accident and this effectively helps in the management of mass casualty incidents.[3,17] Also, early and rapid hematologic changes caused by over exposure to radiation, for example, neutrophil and lymphocyte depletion and a decrease in $\mathrm{CD} 4^{+} / \mathrm{CD}^{+}$ratio may also help in discriminating exposed and non-exposed individuals and estimate the dose exposed.[18]

Samples after 7 and 14 days were collected for predicting the amount of exposure in the latency period in case of a mass casualty incident wherein victims may not have immediate access to medical care for various reasons until days or even weeks after exposure. Decreased T lymphocyte subset levels (especially CTL) in latency period may predict the severity of exposure.[5]

We observed that leucocyte and lymphocyte counts reached nadir level at one day after TBI similar to other 
studies $[5,16]$. In concordance with the findings by Ossetrova et al., the lymphocyte numbers decreased to $15 \%$ of pre-TBI levels one day after TBI.[19] This rapid depletion may be the result of extreme radiosensitivity of mature lymphocytes.[5]

One day after TBI, the decrease in Th cells was more pronounced than in CTL cells and this resulted in a significant decrease in the $\mathrm{CD} 4^{+} / \mathrm{CD}^{+}$ratio. This finding is also in concordance with the literature and may be used to predict the severity of immunosuppression that may develop in exposed individuals.[20]

After 7 and 14 days, although some amount of recovery was noted in all $\mathrm{T}$ lymphocyte subsets, leucopenia and lymphopenia persisted in all subsets and this findingshows similarity with findings in the literature. [12] In a study by $\mathrm{Hu}$, after $3.3 \mathrm{~Gy}, 60$ days were required for neutrophils to return to $95 \%$ of their normal values and for lymphocytes to return to $55 \%$ of their normal levels.[21] Also, Inoue et al. showed radioresistant stem cell subfraction nine days after exposure to 4-6 Gy in mice.[22] Similarly, we found that 7 and 14 days after 5 Gy TBI, almost $20 \%$ of leucocytes and lymphocytes were radioresistant, possibly by means of radioresistant stem cells. This finding may be used as the rationale to opt for cytokine therapy after exposure to IR and the explanation of hematological recovery seen in these individuals. Conversely, this result may inform us about the need for the prolonged use of hematopoietic growth factors, blood product supplies, and antibiotherapy in case of nuclear accidents.[20]

In the latency period, recovery in CTL was slower; 7 days after TBI, Th levels increased by 2.7 times, but CTL levels increased by only 1.007 times. This result reflected an increase in $\mathrm{CD}^{+} / \mathrm{CD}^{+}$ratio, almost reaching the baseline level. Thus, $\mathrm{CD} 4^{+} / \mathrm{CD} 8^{+}$ratio in the latency period may be insignificant in estimating the severity of exposure, and persistently low CTL level, a finding similar to that in our study, may be more predictive.[5]

The regenerative capacity of Treg cells in the latency period was higher than in all other subsets; at the end of the study, Treg levels almost reached half of the preTBI levels. This rapid recovery of Treg cells may have further suppressed Th and CTL recovery.

Data from in-vitro and in-vivo studies showed that Treg cells were radioresistant, although the underlying mechanism and dependency on dose and fractionation are not fully understood.[7,9-11] Intrinsic radioresistance, radiation-induced activation of TGF- $\beta$ promoting Treg cells or the increased output of Treg cells from thymus and spleen, or the increased functional activation of Treg cells in response to local IR or TBI are possible mechanisms. $[9,11]$ In this study, we showed that Treg cells were radiosensitive but their replicative capacity was high and 5 Gy TBI was not enough to suppress Treg cells. Our finding about the radioresistance of Treg cells has two opposing effects. First it makes host favorable for tolerance after TBI to avoid rejection which is desirable. On the other hand it has a negative impact on tumor immunity in terms of tumor control which is an undesirable outcome. $[7,10]$

Radiotherapy (RT) has the reputation of being immunosuppressive. [23,24] But it has recently been established that RT has a dual effect on tumor immunity in the tumor microenvironment; while increasing immunostimulation by the infiltration of CTL and natural killer (NK) cells which inhibit tumor growth on one hand, on the other hand it causes immunosuppression by Treg, tumor-associated macrophages, and myeloidderived suppressor cell infiltration that may result in tumor growth.[11,23-25] Related to this subject, we have found that immunosuppressive Treg cell number significantly decreased one day after TBI, but their selfrenewal was faster than that of Th cells and CTL. This effect may result in additional suppressive effect on antitumor immunity and generalized immunosuppression in case of TBI. Also, single fraction 5 Gy high IR dose may have caused an increase in circulating Treg cells, which is also depicted in literature.[10] These results may warn us in clinical studies about the use, dose, fractionation, technique, timing, and sequencing of RT adjunct to antiCTLA-4 and anti-PD-L1 immunotherapy.[23,25-27] In the future, the immunosuppressive effect of Treg cells on tumor immunity may be overcome by using monoclonal antibodies targeting CD25 as a part of multimodality therapy concurrent with RT and tumor vaccines; in an animal model, Treg cell depletion reportedly resulted in tumor rejection and longer tumor immunity in numerous tumor-inovulated mice. $[9,11,28]$

\section{Limitations of the Study}

A major limitation of this study is the unavailability of multiple blood samples in shorter intervals, the use range of doses, and different dose rates. The absence of sham-irradiated group and the repeating of experiment to show the reproducibility are the other limitations of our study. Also, caution is warranted in the interpretation of these results because the lysis method used to remove red blood cells prior to immunophenotypic analysis of white blood cells may have had an influence on the results.[29]

The relatively higher number of animals evaluated in our experiment is the strength of our study. In ad- 
dition, we preferred blood samples from rats instead of patients treated with TBI to exclude the effects of cancer and previous chemotherapy on the immune system.

\section{Conclusion}

Radiation-induced immunodeficiency may not be explained only by the decrease in lymphocyte cell number but also by the relative increase in Treg cell number that may present an additional role.

In spite of the underpowered nature of our study, our findings might represent an empirical approach by using lymphocyte subset counts as a biodosimetry to predict the severity of the exposed radiation dose.

The next step for us may be the use of other established serum protein combinations in addition to lymphocyte counts, such as acute-phase C-reactive protein, serum amylase activity, Flt3L, p53, p21, IL-6 to create a multiparametric radiation model for biodosimetry. Also, attempts to find out whether there is any functional change in Treg cells induced by IR should be evaluated.

Peer-review: Externally peer-reviewed.

Conflict of Interest: The authors declare that there is no conflict of interest.

Financial Support: None.

Acknowledgments: We thank Ege University Immunology Laboratory and Laboratory Animals Research and Zafer Karaguler for their valuable contributions to our study.

Authorship contributions: Everyone who is listed as an author in this article has made a substantial, direct, intellectual contribution to the work and takes public responsibility for it.

\section{References}

1. Ossetrova NI, Condliffe DP, Ney PH, Krasnopolsky K, Hieber KP, Rahman A, et al. Early-response biomarkers for assessment of radiation exposure in a mouse total-body irradiation model. Health Phys 2014;106(6):772-86.

2. Hall EJ. Acute effects of total-body irradiation. In: Hall EJ, editor. Radiobiology for the Radiologist. Philadelphia, PA: Lippincott Williams \& Wilkins; 2000:12435.

3. Goans RE, Holloway EC, Berger ME, Ricks RC. Early dose assessment in criticality accidents. Health Phys 2001;81(4):446-9.

4. IAEA. Biological Dosimetry. In: Cytogenetic Analysis
For Radiation Dose Assessment: A Manual. Vienna: International Atomic Energy Agency; 2001.

5. Hu S, Smirnova OA, Cucinotta FA. A biomathematical model of lymphopoiesis following severe radiation accidents-potential use for dose assessment. Health Phys 2012;102(4):425-36.

6. Pecaut MJ, Nelson GA, Gridley DS. Dose and dose rate effects of whole-body gamma-irradiation: I. Lymphocytes and lymphoid organs. In Vivo 2001;15(3):195208.

7. Qu Y, Zhang B, Liu S, Zhang A, Wu T, Zhao Y. 2-Gy whole-body irradiation significantly alters the balance of CD4+ CD25- T effector cells and CD4+ CD25+ Foxp3+ T regulatory cells in mice. Cell Mol Immunol 2010;7(6):419-27.

8. Nishikawa H, Sakaguchi S. Regulatory T cells in cancer immunotherapy. Curr Opin Immunol 2014;27:1-7.

9. Qu Y, Jin S, Zhang A, Zhang B, Shi X, Wang J, et al. Gamma-ray resistance of regulatory CD4+CD25+Foxp3+ $\mathrm{T}$ cells in mice. Radiat Res 2010;173(2):148-57.

10. Schaue D, Ratikan JA, Iwamoto KS, McBride WH. Maximizing tumor immunity with fractionated radiation. Int J Radiat Oncol Biol Phys 2012;83(4):1306-10.

11. Kachikwu EL, Iwamoto KS, Liao YP, DeMarco JJ, Agazaryan N, Economou JS, et al. Radiation enhances regulatory $\mathrm{T}$ cell representation. Int J Radiat Oncol Biol Phys 2011;81(4):1128-35.

12. Kusunoki Y, Kyoizumi S, Hirai Y, Suzuki T, Nakashima E, Kodama K, et al. Flow cytometry measurements of subsets of T, B and NK cells in peripheral blood lymphocytes of atomic bomb survivors. Radiat Res 1998;150(2):227-36.

13. Frey O, Bräuer R. Regulatory T cells: magic bullets for immunotherapy? Arch Immunol Ther Exp (Warsz) 2006;54(1):33-43.

14.Zeman E. The Biological Bases of Radiation Oncology. In: Gunderson L, editor. Clinical Radiation Oncology. Elsevier: Phliedelphia; 2016. p. 30.

15. Vodicka I, Hanus J, Hradil J. Use of the adaptive classifier for determination of LD50 in the acute radiation disease. Radiobiol Radiother (Berl) 1989;30(2):143-9.

16. Ossetrova NI, Sandgren DJ, Blakely WF. Protein biomarkers for enhancement of radiation dose and injury assessment in nonhuman primate total-body irradiation model. Radiat Prot Dosimetry 2014;159(1-4):6176.

17. Goans RE, Holloway EC, Berger ME, Ricks RC. Early dose assessment following severe radiation accidents. Health Phys 1997;72(4):513-8.

18. Blakely WF, Salter CA, Prasanna PG. Early-response biological dosimetry-recommended countermeasure enhancements for mass-casualty radiological incidents and terrorism. Health Phys 2005;89(5):494-504. 19. Ossetrova NI, Sandgren DJ, Gallego S, Blakely WF. 
Combined approach of hematological biomarkers and plasma protein SAA for improvement of radiation dose assessment triage in biodosimetry applications. Health Phys 2010;98(2):204-8.

20. Dainiak N, Waselenko JK, Armitage JO, MacVittie TJ, Farese AM. The hematologist and radiation casualties. Hematology Am Soc Hematol Educ Program 2003:473-96.

21. Hu S, Cucinotta FA. Characterization of the radiation-damaged precursor cells in bone marrow based on modeling of the peripheral blood granulocytes response. Health Phys 2011;101(1):67-78.

22. Inoue T, Hirabayashi Y, Mitsui H, Sasaki H, Cronkite EP, Bullis JE Jr, et al. Survival of spleen colony-forming units (CFU-S) of irradiated bone marrow cells in mice: evidence for the existence of a radioresistant subfraction. Exp Hematol 1995;23(12):1296-300.

23. Derer A, Frey B, Fietkau R, Gaipl US. Immune-modulating properties of ionizing radiation: rationale for the treatment of cancer by combination radiotherapy and immune checkpoint inhibitors. Cancer Immunol Immunother 2016;65(7):779-86.

24. Anacak Y, Kamer S, Sert F. Combining radiotherapy with immunotherapy. Marmara Med J 2015;28(Special
Issue 1):40-4.

25. Walle T, Martinez Monge R, Cerwenka A, Ajona D, Melero I, Lecanda F. Radiation effects on antitumor immune responses: current perspectives and challenges. Ther Adv Med Oncol 2018;10:1758834017742575.

26. Twyman-Saint Victor C, Rech AJ, Maity A, Rengan R, Pauken KE, Stelekati E, et al. Radiation and dual checkpoint blockade activate non-redundant immune mechanisms in cancer. Nature 2015;520(7547):373-7.

27. Barker CA, Postow MA. Combinations of radiation therapy and immunotherapy for melanoma: a review of clinical outcomes. Int J Radiat Oncol Biol Phys 2014;88(5):986-97.

28. Kudo-Saito C, Schlom J, Camphausen K, Coleman $\mathrm{CN}$, Hodge JW. The requirement of multimodal therapy (vaccine, local tumor radiation, and reduction of suppressor cells) to eliminate established tumors. Clin Cancer Res 2005;11(12):4533-44.

29. Einwallner E, Subasic A, Strasser A, Augustin D, Thalhammer R, Steiner I, et al. Lysis matters: red cell lysis with FACS Lyse affects the flow cytometric enumeration of circulating leukemic blasts. J Immunol Methods 2013;390(1-2):127-32. 